\title{
PRONÓSTICOS BAYESIANOS PARA REPUESTOS DE AUTOMÓVILES USANDO SIMULACIÓN ESTOCÁSTICA
}

\author{
BAYESIAN FORECASTINGS FOR AUTOMOBILE PARTS \\ USING STOCHASTIC SIMULATION
}

\author{
David F. Muñoz Negrón ${ }^{1}$ y Diego F. Muñoz Medina²
}

\section{RESUMEN}

Este artículo presenta el desarrollo y la aplicación de un modelo de simulación que fue utilizado para pronosticar la demanda de repuestos de automóviles a partir de información obtenida de un distribuidor de automóviles y repuestos en México, D. F. En particular, este trabajo ilustra, con un modelo sencillo, cómo se pueden combinar la simulación estocástica y la estadística bayesiana para modelar y resolver problemas complejos de pronóstico. El marco propuesto es suficientemente general para aplicarse a modelos muy detallados del fenómeno en estudio. Los resultados obtenidos demuestran cómo se puede incorporar la incertidumbre en los parámetros del modelo, y su aplicación usando datos reales, revela cómo la amplitud de la muestra produce una distribución posterior con poca influencia sobre la distribución a priori.

Palabras claves: pronósticos, pronóstico de repuestos, estimación bayesiana, puntos de reorden, nivel de servicio.

\begin{abstract}
This article presents the development and application of a simulation model that was used to forecast the demand of automobile parts using information from a car dealer in Mexico, D. F. In particular, this work illustrates, using a simple model, how stochastic simulation and Bayesian statistics can be combined to model and solve complex forecasting problems. The proposed framework is general enough to be applied to very detailed models of the system under study. The results obtained demonstrate how uncertainty on the parameters of the model can be incorporated, and the application using real data shows how a large sample size produces a posterior distribution that has little influence from the prior distribution.
\end{abstract}

Keywords: Forecasts, repair forecasts, Bayesian inferences, reorder points, level of service.

1. Instituto Tecnológico Autónomo de México. Ph. D. en Investigación de Operaciones de Stanford University, California. <davidm@itam.mx>.

2. Estudiante del Master of Science en Management Science and Engineering, Stanford University. Ingeniero industrial, Instituto Tecnológico Autónomo de México (México). <dkedmun@stanford.edu>. 


\section{INTRODUCCIÓN}

El pronóstico de la demanda juega un papel fundamental en la estrategia de numerosas organizaciones de manufactura o servicios. La elaboración de pronósticos es de gran ayuda en la programación de la mano de obra, la obtención de niveles de servicio adecuados y la determinación de los requerimientos de recursos, entre otras aplicaciones (Makridakis et ál., 1998). La capacidad para elaborar pronósticos con un alto grado de precisión es un objetivo que adquiere creciente importancia en un gran número de empresas $y$, en particular, juega un papel fundamental para pronosticar demandas que tienen un patrón esporádico.

Diversos autores como Wacker y Sprague (1998), y Zotteri y Kalchschmidt (2007) consideran que la precisión de un pronóstico depende, sensiblemente, de la técnica cuantitativa que se emplea para elaborarlo. Por lo tanto, este artículo ha sido motivado por la necesidad de formular y aplicar nuevas herramientas para la elaboración de pronósticos de la demanda y, en particular, para los casos en los que la demanda es esporádica. De acuerdo con el trabajo de Caniato et ál. (2005) y Kalchschmidt et ál. (2006), es indispensable proponer técnicas de pronósticos que no solo tomen en cuenta la serie de tiempo, sino también la estructura del proceso que genera la demanda (variabilidad no sistemática). Por esta razón, el presente artículo mostrará cómo se puede aplicar técnicas de simulación y estadística bayesiana en un modelo que toma en cuenta las características específicas del sistema que se pretende estudiar.

En la práctica, un modelo de pronóstico puede llegar a ser complejo, en el sentido que no es posible obtener expresiones analíticas para los estimadores puntuales y de variabilidad requeridos para el pronóstico. A manera de ejemplo, puede mencionarse el modelo de Croston (1972) propuesto para pronosticar demanda intermitente, cuyos resultados fueron corregidos por Rao (1973) y, más adelante, por Syntetos y Boylan (2001). Por esta razón es relevante proponer metodologías que permitan incorporar un modelo complejo de pronóstico utilizando la simulación para estimar los parámetros necesarios para construir el pronóstico. Curiosamente, hasta el día de hoy se había estado utilizando simulación para comparar el desempeño de diferentes técnicas de pronósticos (véase Bartezzaghi et ál., 1999; Zotteri \& Kalchschmidt, 2007a y b). Sin embargo, la literatura sobre el uso de la simulación como herramienta para construir pronósticos todavía es escasa. Una de estas pocas referencias es Willemain et ál. (2004), donde se utiliza un modelo de simulación para pronosticar demandas con patrones intermitentes y se obtiene mejores resultados que con los métodos de Croston y de suavizamiento exponencial, aunque en este trabajo no se incorporó la incertidumbre paramétrica en el pronóstico.

Es conveniente remarcar las ventajas que presenta el enfoque bayesiano usado en este artículo, en comparación con las alternativas tradicionales ("frecuentistas"), cuando se utiliza un modelo complejo para construir pronósticos. En primer lugar, en las aplicaciones tradicionales donde se utiliza la simulación estocástica como herramienta de análisis se suele fijar el valor de los parámetros de las distribuciones de probabilidad (reemplazándolos, por ejemplo, por estimadores de máxima verosimilitud); en este caso, el método tradicional no es capaz de incorporar la incertidumbre paramétrica en el pronóstico y puede subestimar el riesgo del mismo. Por otro lado, si bien existen métodos tradicionales que consideran la incertidumbre paramétrica, en algún momento se ven forzados a sustituir el valor del parámetro por un estimador puntual; por ejemplo, Cheng y Holland (2004) proponen que al utilizar simulación estocástica, la incertidumbre paramétrica puede incorporarse utilizando bootstrap paramétrico, que consiste en remuestrear (a través de simulación) los estimadores de máxima verosimilitud de la distribución de probabilidades que se obtiene al reemplazar el valor de los parámetros por el estimador máximo verosímil que se calculó a partir de una muestra de observaciones (reales). En cambio, como se ilustra en este artículo, un enfoque bayesiano permite incorporar la incertidumbre paramétrica de manera natural, sin necesidad de asumir algún valor particular para los parámetros.

Debido a las consideraciones mencionadas, la construcción de pronósticos bajo un enfoque bayesiano es aconsejable cuando el investigador está interesado en cuantificar la incertidumbre paramétrica (por ejemplo, 
porque los datos provienen de muestras de un pequeño tamaño), como se ilustra en Alba y Mendoza (2007), donde los autores presentan la aplicación de un método bayesiano para datos estacionales y muestran que este método se desempeña mejor que los de series de tiempo tradicionales cuando la serie de tiempo es corta.

Este artículo presenta el desarrollo y la aplicación de un modelo de simulación que fue utilizado para pronosticar la demanda de repuestos de automóviles que experimenta un concesionario en México, D. F. Asimismo, se ilustra el potencial de la simulación como una poderosa herramienta para elaborar pronósticos utilizando un modelo complejo del sistema en estudio. La metodología utilizada puede ser vista como un ejemplo de cómo es posible atacar problemas de pronóstico cuando la complejidad del modelo no permite obtener expresiones analíticas para los estimadores de pronóstico puntual y de la incertidumbre en el pronóstico.

El artículo se compone de cuatro secciones. En la primera sección se presenta un marco teórico general para la aplicación de la simulación y la estadística bayesiana para construir pronósticos y se revisan las técnicas disponibles para producir los estimadores de los parámetros requeridos para pronosticar. En la segunda sección se presentan dos modelos simples que pueden ser utilizados para producir pronósticos de repuestos que siguen un patrón de demanda esporádica. En la tercera sección se utilizan datos reales de la demanda de embragues, obtenidos de un distribuidor de automóviles y repuestos en México, D. F., para implementar uno de los modelos descritos en la segunda sección con las técnicas de simulación presentadas en la primera sección. En particular, se ilustra cómo se estiman la demanda esperada y el punto de reorden para la demanda de embragues durante el tiempo de demora de una orden de suministro. Finalmente, en la cuarta sección se presentan las principales conclusiones de este trabajo y se discuten direcciones para futuras investigaciones.

\section{DEFINICIÓN DEL PROBLEMA}

En esta sección se hace una pequeña revisión del marco y de las técnicas presentadas en Muñoz (2009), que serán aplicadas en este artículo. En dicho artículo se proponen dos pasos esenciales para la construcción de pronósticos utilizando simulación. El primer paso consiste en la evaluación de la incertidumbre sobre los parámetros del modelo utilizando la información disponible $(x)$ y una densidad a priori $p(\theta)$. En el segundo paso se utilizan el modelo de simulación y la densidad posterior para estimar los parámetros requeridos para hacer pronósticos sobre la variable de respuesta.

La densidad a priori $p(\theta)$ refleja la incertidumbre inicial sobre el vector de parámetros $\Theta$, y existen fundamentalmente dos puntos de vista para proponer $p(\theta)$. El primero consiste en usar una densidad a priori no informativa, la cual es apropiada cuando se desea que no se "favorezca" ningún valor posible de $\Theta$ sobre otro, y este constituye un punto de vista "objetivo" (una reciente discusión sobre este tema puede verse en Berger et ál., 2009). Las densidades a priori no informativas han sido muy estudiadas, y varios libros sobre estadística bayesiana (por ejemplo, Bernardo \& Smith, 2000) publican la correspondiente densidad a priori no informativa (de referencia) para las distribuciones más usadas. El segundo enfoque es un punto de vista "subjetivo" y consiste en establecer la densidad a priori con base en opiniones de expertos, véase por ejemplo, Kraan y Bedford (2005) para una discusión sobre la construcción de una densidad a priori con base en pronósticos de expertos.

Luego de identificar la densidad a priori $p(\theta)$, la incertidumbre paramétrica se cuantifica por medio de la densidad posterior $p(\theta \mid x)$ :

$$
p(\theta \mid x)=\frac{p(\theta) L(x \mid \theta)}{\int_{P} p(\theta) L(x \mid \theta) d \theta}
$$

para $x \in \mathfrak{R}^{n}$ y $\theta \in P$, donde $L(x \mid \theta)$ es la función de verosimilitud, que para el caso particular en que $x=\left(x_{1}, x_{2}, \ldots, x_{n}\right)$ es un conjunto de observaciones de una muestra aleatoria $X=\left(X_{1}, X_{2}, \ldots, X_{n}\right)$ de una función de densidad $f(y \mid \theta)$, la función de verosimilitud toma la forma:

$$
L(x \mid \theta)=f\left(x_{1} \mid \theta\right) f\left(x_{2} \mid \theta\right) \ldots f\left(x_{n} \mid \theta\right)
$$


En general, un pronóstico para la variable de respuesta $W$ queda definido por su función de distribución acumulada (f.d.a.) $F(w \mid x)=P[W \leq w \mid X=x]$. Sin embargo, desde un punto de vista práctico, un pronóstico se expresa en términos de un pronóstico puntual y una medida de la incertidumbre de dicha estimación. El pronóstico puntual más usado en estadística bayesiana es la esperanza:

$$
r(x)=E[W \mid X=x]
$$

Otra medida de desempeño de importancia práctica es el $\alpha$-cuantil definido por:

$$
q_{\alpha}(x)=\inf \{w: F(w \mid x) \geq \alpha\}
$$

para $0<\alpha<1$. Los $\alpha$-cuantiles son útiles para evaluar la incertidumbre del pronóstico puntual $r(x)$, ya que permiten construir un intervalo de predicción del $(1-\beta) 100 \%$ (donde $0<\beta<1$ ), que toma la forma de $\left[q_{\beta / 2}(x), q_{1-\beta / 2}(x)\right]$. Dicho intervalo es llamado un intervalo de predicción del $(1-\beta) 100 \%$ porque $P\left[q_{\beta / 2}(x) \leq W \leq q_{1-\beta / 2}(x) \mid X=x\right]=1-\beta$, siempre y cuando $F(w \mid x)$ sea continua en $q_{\beta / 2}(x)$ y $q_{1-\beta / 2}(x)$.

Los cuantiles también son útiles para calcular puntos de reorden para la administración de inventarios. En este caso, cuando $W$ es la demanda durante el tiempo de demora del suministro, el $\alpha$-cuantil $q_{\alpha}(x)$ puede ser interpretado como el punto de reorden para alcanzar un nivel de servicio (tipo-I) del $100 \alpha \%$ (véase, por ejemplo, Chopra \& Meindl, 2004).

Cuando las expresiones analíticas para los parámetros del pronóstico definidas en las ecuaciones (3) y (4) no pueden ser obtenidas (o es muy complicado hacerlo), se puede hacer una estimación de estos parámetros utilizando simulación. En el gráfico 2 de Muñoz $(2009$, p. 15) se presenta un primer algoritmo, que será llamado muestreo posterior (MP), debido a que se generan valores del parámetro $\Theta$ a partir de la densidad posterior $p(\theta \mid x)$ para luego obtener observaciones independientes e idénticamente distribuidas de la variable de respuesta $W$ que permiten estimar el pronóstico puntual $r(x)$ y el $\alpha$-cuantil $q_{\alpha}(x)$. Como es bien sabido, dichos estimadores son consistentes, lo cual implica que se aproximan al parámetro a medida que se incre- menta el número de repeticiones $m$. Sin embargo, cabe mencionar que el número de repeticiones necesarias para alcanzar un nivel de precisión predeterminado depende en gran medida del problema. Por lo tanto, es una buena práctica calcular una medida de precisión del estimador puntual e incrementar las réplicas del experimento hasta obtener la precisión deseada.

El procedimiento más difundido para evaluar la precisión de un estimador puntual obtenido por medio de simulación consiste en calcular el ancho medio de un intervalo de confianza (IC) asintótico. El ancho medio de un IC de 100(1 - $\beta) \%$ de confianza apropiado para el estimador $\hat{r}(x)$ obtenido por MP está dado por:

$$
H_{\beta}[\hat{r}(x)]=z_{1-\beta / 2} \frac{S(x)}{\sqrt{m}}
$$

donde $S(x)=m^{-1 / 2} \sqrt{\left[W_{i}-\hat{r}(x)\right]^{2}}$ y $z_{1-\beta / 2}$ denota el $(1-\beta / 2)$-cuantil de una distribución normal estándar. Una interpretación simple de un ancho medio es que el parámetro $r(x)$ se halla entre $\hat{r}(x) \pm H_{\beta}[\hat{r}(x)]$ con una confianza del $100(1-\beta) \%$. Por ello, un ancho medio resulta muy útil para evaluar la precisión de un estimador puntual. Análogamente, un ancho medio del $100(1-\beta) \%$ de confianza para el estimador $\hat{q}_{\alpha}(x)$ obtenido a través del MP está dado por:

$$
H_{\beta}\left[\hat{q}_{\alpha}(x)\right]=\left(Y_{n_{1}}+Y_{n_{2}}\right) / 2
$$

donde $Y_{1} \leq Y_{2} \leq \ldots \leq Y_{n}$ resultan de ordenar las $W_{i}, \quad n_{1}=\left[m \alpha-z_{1-\beta / 2}[m \alpha(1-\alpha)]^{1 / 2}\right\rfloor, \mathrm{y}$ $n_{2}=\left\lceil m \alpha+z_{1-\beta / 2}[m \alpha(1-\alpha)]^{1 / 2}\right\rceil$. Una prueba de la validez asintótica de este ancho medio se puede hallar en Serfling (1980).

La técnica clásica para analizar experimentos por simulación transitoria adopta un algoritmo muy similar al algoritmo MP, con la diferencia de que el valor de $\Theta$ es fijo y, por ello, no se requiere el muestreo de $p(\theta \mid x)$. Por lo tanto, la varianza de la variable de respuesta bajo un enfoque clásico toma la forma:

$$
\begin{gathered}
\sigma^{2}(\theta)=E\left[W^{2} \mid X=x, \Theta=\theta\right]- \\
(E[W \mid X=x, \Theta=\theta])^{2}
\end{gathered}
$$


donde $\theta \in P$ es el valor que se ha fijado para $\Theta$. Por otro lado, bajo el enfoque bayesiano, la varianza de la variable de respuesta es:

$$
\sigma_{W}^{2}=E\left[W^{2} \mid X=x\right]-(E[W \mid X=x])^{2}
$$

de manera que sumando y restando el término $E\left[r_{l}^{2}(\Theta) \mid X=x\right]$ en (8), se puede verificar que:

$$
\sigma_{W}^{2}=\sigma_{p}^{2}+\sigma_{s}^{2}
$$

donde $\sigma_{p}^{2}=E\left[r_{1}^{2}(\Theta) \mid X=x\right]-\left(E\left[r_{1}(\Theta) \mid X=x\right]\right)^{2}$, $r_{l}(\theta)=E[W \mid X=x, \Theta=\theta], \sigma_{s}^{2}=E\left[\sigma^{2}(\Theta) \mid X=x\right]$, y $\sigma^{2}(\theta)$ está definido en (7). Nótese que $\sigma_{p}^{2}=0$ y $\sigma_{s}^{2}$ $=\sigma^{2}(\theta)$ bajo un enfoque tradicional, razón por la cual $\sigma_{p}^{2}$ es llamada la varianza paramétrica y $\sigma_{s}^{2}$ es llamada la varianza estocástica.

Para implementar el algoritmo MP, se requiere de un método para generar muestras de la densidad posterior $p(\theta \mid x)$, el cual puede estar disponible si se ha identificado la familia de distribuciones correspondiente a $p(\theta \mid x)$. Sin embargo, en muchas situaciones puede ser difícil obtener una expresión analítica que permita identificar esta familia de distribuciones. En este caso, se puede aplicar una técnica conocida como la cadena de Markov Monte Carlo (CMMC), la cual no requiere de un algoritmo que genere muestras de $p(\theta \mid x)$.

En este artículo se aplicará el algoritmo del gráfico 4 de Muñoz (2009, p. 19), que es una implementación de la CMMC conocida como el muestreador independiente, pues deben generarse muestras de una densidad auxiliar $q(\theta)$ (donde $q(\theta)>0$ cuando $p(\theta)>0$ ). Diversos autores (por ejemplo, Asmussen \& Glynn, 2007) concuerdan en que este algoritmo se desempeña mejor cuando $q(\theta)$ es parecida a la densidad posterior $p(\theta \mid x)$.

Nótese que, a pesar de que no es necesario para calcular los estimadores puntuales, el algoritmo CMMC por utilizar divide el número de réplicas $m$ en $b$ grupos de longitud $m_{b}$. Esto porque se propone el uso del método de promedios por grupos para producir intervalos de confianza asintóticos para los estimadores puntuales, como se explica a continuación.
Habiendo escogido un número $b$ de grupos entre 5 y 20 (como sugiere Schmeiser, 1982), los siguientes anchos medios del $100(1-\beta) \%$ de confianza son asintóticamente válidos (a medida que $m \rightarrow \infty$ ) para $r(x)$ y $q_{\alpha}(x)$, respectivamente:

$$
H_{\beta}\left[\hat{r}_{M C}(x)\right]=t_{(b-1,1-\beta / 2)} \frac{S_{M C}(x)}{\sqrt{b}} \quad \mathrm{y}
$$

$$
H_{\beta}\left[\hat{q}^{M C}(x)\right]=t_{(b-1,1-\beta / 2)} \frac{S^{M C}(x)}{\sqrt{b}}
$$

donde $t_{(b-1,1-\beta / 2)}$ denota el $(1-\beta / 2)$-cuantil de una distribución t-Student con $(b-1)$ grados de libertad, $\hat{r}_{M C}(x), \hat{q}^{M C}(x)$ son los estimadores puntuales del algoritmo CMMC, $\mathrm{y}$ :

$$
\begin{gathered}
S_{M C}(x)=\sqrt{\frac{\sum_{i=1}^{b}\left[\hat{r}_{i}-\hat{r}_{M C}(x)\right]^{2}}{b-1}} \\
S_{\alpha}^{M C}(x)=\sqrt{\frac{\sum_{i=1}^{b}\left[\hat{q}_{\alpha}^{i}-\bar{q}_{\alpha}\right]^{2}}{b-1}}
\end{gathered}
$$

donde $\hat{r}_{i}$ y $\hat{q}^{i}$ son los estimadores por grupos definidos en el algoritmo CMMC, y $\bar{q}_{\alpha}=b^{-1} \sum_{i=1}^{b} \hat{q}_{\alpha}^{i}$. Para una discusión sobre la validez de los intervalos de confianza presentados en las ecuaciones (10) y (11), se puede consultar Muñoz (2009, p. 20).

Como ya se mencionó, el muestreador independiente presenta un mejor desempeño cuando $q(\theta)$ es cercana a la densidad posterior $p(\theta \mid x)$. Por ello, puede proponerse un procedimiento para seleccionar la densidad $q(\theta)$ tomando en cuenta que, bajo condiciones de regularidad, una densidad posterior satisface un teorema de límite central (a medida que el tamaño de muestra $n \rightarrow \infty$ ), donde la distribución límite es una normal (multivariada). Por lo tanto, una propuesta razonable para $q(\theta)$ es una densidad correspondiente a una distribución normal multivariada con un vector 
de medias $\mu_{n}$ y una matriz varianza-covarianza $V_{n}$, donde los parámetros $\mu_{n}$ y $V_{n}$ pueden determinarse de acuerdo con las características particulares de $p(\theta \mid x)$.

Los siguientes pasos para seleccionar los parámetros $\mu_{n}$ y $V_{n}$ están basados en el teorema 5.14 de Bernardo y Smith (2000), y pueden ser implementados cuando la función de verosimilitud tiene la forma que se presenta en la ecuación (2).

1. Hacer $\mu_{n}$ igual al vector que minimiza $L_{n}(\theta) \stackrel{\text { def }}{=} \log [p(x \mid \theta)]$, resolviendo $=\left.\nabla L_{n}(\theta)\right|_{\theta=\mu_{n}}=0$.

2. Hacer $V_{n}=\left[-L_{n} "\left(\mu_{n}\right)\right]^{-1}$, donde $L_{n}{ }^{\prime \prime}\left(\mu_{n}\right) \stackrel{\operatorname{def}}{=}\left(\frac{\partial^{2} L_{n}(\theta)}{\partial \theta_{i} \partial \theta_{j}}\right) \mid \theta=\mu_{n}$ es la matriz Hessiana, la cual tiene que ser positiva definida para que este procedimiento sea válido.

En la tercera sección se presenta un ejemplo sencillo de la implementación de este procedimiento para proponer una distribución de muestreo $q(\theta)$ para el muestreador independiente.

\section{MODELOS DE PRONÓSTICO PARA DEMANDA DE REPUESTOS}

En esta sección se presentan dos modelos sencillos que pueden usarse para pronosticar la demanda de repuestos, y serán útiles para ilustrar cómo se pueden aplicar las técnicas mencionadas en la sección anterior. En ambos modelos se asume que las fallas ocurren aleatoriamente, y la única diferencia radica en la forma en que están disponibles los datos, como se explica a continuación.

En ambos modelos se tienen $k$ máquinas durante el periodo de pronóstico, y las fallas ocurren independientemente con la misma tasa $\Theta \in P=(0, \infty)$ para cada máquina. Existe incertidumbre en la tasa de fallas $\Theta$, reflejada en la densidad a priori $p(\theta)$, y para $i=1,2, \ldots, k, N_{i}=\left\{N_{i}(t): t \geq 0 ; \Theta\right\}$ denota el proceso de fallas para el componente $i$, los que se asumen condicionalmente independientes dado $\Theta$ (véase la definición, por ejemplo, en Chung, 1974), y:

$$
\begin{aligned}
& P\left[N_{i}(t+s)-N_{i}(t)=\right. \\
& \left.j \mid \Theta, N_{i}(u), 0 \leq u \leq t\right]= \\
& \frac{e^{-\Theta s}(\Theta s)^{j}}{j !}
\end{aligned}
$$

para $j=0,1, \ldots, t, s \geq 0, \quad i=1,2, \ldots, k$, es decir, los procesos de fallas son procesos de Poisson con la misma tasa $\Theta$.

\section{Un modelo con datos de tiempos entre fallas sucesivas}

En este modelo, la información disponible corresponde a los tiempos entre fallas sucesivas de cada parte. En consecuencia, las observaciones provienen de una muestra aleatoria $X=\left(X_{1}, X_{2}, \ldots, X_{n}\right)$ de la distribución exponencial con densidad:

$$
f(y \mid \theta)= \begin{cases}\theta e^{-\theta y}, & y>0, \\ 0, & \text { de otra forma }\end{cases}
$$

y de (2) sigue que la función de verosimilitud está dada por:

$$
L(x \mid \theta)=\theta^{n} e^{-\theta \sum_{i=1}^{n} x_{i}}
$$

Se sabe que la distribución no informativa para una densidad a priori exponencial (véase, por ejemplo, Bernardo \& Smith, 2000) es $p(\theta)=\theta^{-1}$, por lo que sigue de (1) y (12) que:

$$
p(\theta \mid x)=\frac{\left(\sum_{i=1}^{n} x_{i}\right)^{n} \theta^{n-1} e^{-\theta} \sum_{i=1}^{n} x_{i}}{(n-1) !}
$$

expresión que corresponde a una distribución Gama $\left(n, \sum_{i=1}^{n} x_{i}\right)$, donde para $\beta_{1}, \beta_{2}>0, \operatorname{Gama}\left(\beta_{1}, \beta_{2}\right)$ denota la distribución gama con esperanza $\beta_{1} \beta_{2}{ }^{-1}$. 
Se desea pronosticar el número de componentes que fallarán durante un periodo de tiempo de longitud $t_{0}$, por lo que la variable de respuesta a pronosticar es:

$$
W=\sum_{i=1}^{k} N_{i}\left(t_{0}\right)
$$

dado $[X=x]$.

Con la finalidad de obtener una expresión analítica para $r(x)$, se puede aplicar la Proposición 1 de Muñoz (2009, p. 11), teniendo en cuenta que $r_{l}(\theta)=E[W \mid \Theta$ $=\theta]=k t_{0} \theta$, se obtiene:

$$
r(x)=\int_{0}^{\infty} r_{l}(\theta) p(\theta \mid x) d \theta=k t_{0} n\left(\sum_{i=1}^{n} x_{i}\right)^{-1}
$$

Para el caso en que $t_{0}$ corresponde al tiempo de demora de una orden, el punto de reorden para un nivel de servicio tipo-I del $100 \alpha \%$ es $q_{\alpha}(x)$, definido en la ecuación (4). Debido a que no se dispone de una expresión analítica sencilla para este parámetro, el uso del algoritmo MP puede ser apropiado para estimar $q_{\alpha}(x)$ usando simulación.

Supóngase ahora que se tienen $Q$ unidades del repuesto en inventario al inicio de un periodo de longitud $t_{0}$. En este caso, dos medidas de desempeño de importancia para la administración de inventarios son el nivel de servicio tipo-I (100 $\left.\alpha_{1} \%\right)$, y el nivel de servicio tipo-II (100 $\left.\alpha_{2} \%\right)$, donde:

$$
\begin{gathered}
\alpha_{1}=P[W \leq Q \mid X=x] \quad y \\
\alpha_{2}=E[\min \{1, Q / W\} \mid X=x]
\end{gathered}
$$

Como puede apreciarse de estas ecuaciones, ambas medidas de desempeño tienen la misma forma de la esperanza $r(x)$, para una variable de respuesta apropiadamente definida. Nuevamente, obtener expresiones analíticas para estas medidas de desempeño no es una tarea fácil, por lo que se justifica la aplicación del algoritmo MP para estimar $\alpha_{1}$ y $\alpha_{2}$.

\section{Un modelo con datos de censo}

Supóngase que no se tienen registros de los tiempos entre fallas sucesivas, pero en cambio, para cada periodo de tiempo $i=1,2, \ldots, p$, se ha registrado el número $k_{i}$ de máquinas en operación durante el periodo $i$, y el número de fallas por máquina durante el periodo $i$. En este caso, los datos toman la forma de $x=\left(x_{11}, \ldots, x_{1 k_{1}}, \ldots, x_{p l}, \ldots, x_{p k_{p}}\right)$, donde $x_{i j}$ es el número de fallas de la $j$-ésima máquina durante el periodo $i, j=1,2, \ldots, k_{i} ; i=1,2, \ldots, p$ (en este caso $n=\sum_{i=1}^{p} k_{i}$ ).

Para simplificar la notación, se asume que cada periodo tiene la longitud de una unidad de tiempo, lo que se logra expresando la tasa de fallas en la escala apropiada (fallas por unidad de tiempo). Como los procesos de fallas se asumen condicionalmente independientes dado $\Theta$, el vector de datos $x$ puede considerarse como el conjunto de observaciones de una muestra aleatoria $X=\left(X_{1}, X_{2}, \ldots, X_{n}\right)$ de una distribución de Poisson, de acuerdo con una función de probabilidades (discreta):

$$
f(y \mid \theta)=\frac{e^{-\theta} \theta^{y}}{y !} \quad y=0,1, \ldots,
$$

y sigue de (2) que la función de verosimilitud resulta:

$$
L(x \mid \theta)=\frac{e^{-\theta \sum_{i=1}^{p} k_{i}} \theta \sum_{i=1}^{p} \sum_{j=1}^{k_{i}} x_{i j}}{\coprod_{i=1}^{p} \prod_{j=1}^{k_{i}} x_{i j} !}
$$

Como se sabe (véase, por ejemplo, Bernardo \& Smith, 2000), la densidad a priori objetiva para la distribución de Poisson es $p(\theta)=\theta^{-1 / 2}$, por lo que sigue de (1) y (17) que;

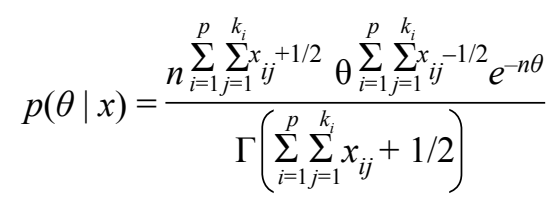

que corresponde a la distribución Gama $\left(\sum_{i=1}^{p} \sum_{j=1}^{k_{i}} x_{i j}+1 / 2, n\right)$.

Como en el modelo anterior, aquí se desea pronosticar el número de componentes que fallan durante un periodo de tiempo de longitud $t_{0}$, por lo que la variable de respuesta obedece a la ecuación (14). Teniendo en cuenta que ahora la densidad posterior $p(\theta \mid x)$ está definida 
en (18), se puede proceder como en la ecuación (15) para obtener una expresión analítica para $r(x)$ :

$$
\begin{aligned}
& r(x)=\int_{0}^{\infty} r_{l}(\theta) p(\theta \mid x) d \theta= \\
& n^{-1} k t_{0}\left(\sum_{i=1}^{p} \sum_{j=1}^{k_{i}} x_{i j}+1 / 2\right)
\end{aligned}
$$

En este caso también el algoritmo MP puede ser utilizado para estimar el punto de reorden $q_{\alpha}(x)$, o el nivel de servicio definido en (16).

Nótese que en los dos modelos presentados no se ha requerido utilizar el algoritmo CMMC, debido a que se ha podido reconocer la familia de distribuciones a la que pertenece la densidad posterior (en ambos casos es gama). Sin embargo, si no se usara la densidad a priori objetiva en estos modelos, pudiera ocurrir que la familia de distribuciones a la que pertenece la densidad posterior $p(\theta \mid x)$ no fuera fácil de reconocer, y en este caso el algoritmo CMMC sería de utilidad, como se ilustra más adelante en el segundo acápite de la siguiente sección.

\section{PRUEBAS EXPERIMENTALES}

En esta sección se aplica el modelo que considera datos de censo para pronosticar la demanda de embragues (de un modelo particular) que experimenta un distribuidor de autos. Se ilustra la aplicación de los métodos descritos en secciones previas utilizando datos de la demanda de embragues de un distribuidor de autos y repuestos en México, D. F.

\section{Aplicación de la técnica muestreo posterior (MP) para calcular puntos de reorden y niveles de servicio}

El modelo de autos $A$ es el modelo de una línea que ha tenido mucha acogida, y que se continúa produciendo y vendiendo actualmente. En el Cuadro 1 se presentan datos disponibles sobre la demanda de este modelo, que incluyen la demanda de autos y de embragues para cada mes del año 2008. Con la finalidad de aplicar el modelo con datos de censo, se asume que los clientes que compran su auto en un distribuidor particular son los mismos clientes que compran los repuestos de embrague en dicho distribuidor. En consecuencia, el número $k_{i}$ de autos en operación al final del periodo $i$ es igual a la cantidad de autos del modelo $A$ vendidos por el distribuidor hasta dicho periodo, de manera que $k_{i}=k_{i-1}+S_{i}$, donde $S_{i}$ es la cantidad de autos vendidos en el periodo $i$. Como puede observarse de los datos del Cuadro 1, el año 2008 empezó con 337 autos en operación del modelo A (de acuerdo con las ventas de los años anteriores).

Cuadro 1. Demanda de Autos y Embragues para el Modelo A en 2008

\begin{tabular}{lccc}
\hline \multicolumn{1}{c}{$\begin{array}{c}\text { Mes } \\
(i)\end{array}$} & $\begin{array}{c}\text { Ventas de } \\
\text { autos } \\
\left(S_{i}\right)\end{array}$ & $\begin{array}{c}\text { Autos en } \\
\text { operación } \\
\left(k_{i}\right)\end{array}$ & $\begin{array}{c}\text { Demanda de } \\
\text { embragues } \\
k_{i} \\
\left(\sum_{j=1} x_{i j}\right)\end{array}$ \\
\hline Enero (1) & 4 & 341 & 3 \\
Febrero (2) & 1 & 342 & 3 \\
Marzo (3) & 6 & 348 & 2 \\
Abril (4) & 9 & 357 & 3 \\
Mayo (5) & 6 & 363 & 3 \\
Junio (6) & 15 & 378 & 3 \\
Julio (7) & 7 & 385 & 3 \\
Agosto (8) & 2 & 387 & 1 \\
Septiembre (9) & 8 & 395 & 4 \\
Octubre (10) & 16 & 411 & 3 \\
Noviembre (11) & 20 & 431 & 3 \\
Diciembre (12) & 15 & 446 & 2 \\
Total & $\mathbf{1 0 9}$ & $\mathbf{4 , 5 8 4}$ & $\mathbf{3 2}$ \\
\hline
\end{tabular}

Fuente: Datos proporcionados por un concesionario de autos en México, D. F.

Se desea pronosticar la demanda $W$, de acuerdo con la ecuación (14), cuando $t_{0}=0.5$ y $k=500$, ya que se asume que la demora de una orden de embragues es de aproximadamente 15 días y existen 500 autos en operación durante el periodo de pronóstico.

Usando (19) se calculó el pronóstico puntual de la demanda de embragues durante la demora del pedido y se obtuvo $r(x)=1.82702$. Posteriormente, se aplicó el algoritmo MP para estimar $q_{0.9}(x)$, el punto de reorden para un nivel de servicio de $90 \%$. Los resultados para la estimación de $r(x)$ y $q_{0.9}(x)$ usando $m=10^{6}$ repeticiones se resumen en el Cuadro 2 . El ancho medio para $r(x)$ indica que el error en la estimación de $\hat{r}(x)$ es menor que 0.00228 , aunque ya sabemos que la diferencia entre $r(x)$ y $\hat{r}(x)$ tiene menos de 4 decimales significativos. 
Cuadro 2. Estimación de Parámetros Usando MP con $m=10^{6}$ Repeticiones

\begin{tabular}{ccc}
\hline Parámetro & Estimación por simulación & Ancho medio \\
\hline$r(x)$ & $\hat{r}(x)=1.82695$ & 0.00228 \\
$q_{0.9}(x)$ & $\hat{q}_{0.9}(x)=4$ & 0.0 \\
\hline
\end{tabular}

En el caso de la estimación de $q_{0.9}(x)$, se puede apreciar del Cuadro 2 que el ancho medio es de 0 , lo cual no es sorprendente si se toma en cuenta que la variable de respuesta $W$ es discreta, y su función de distribución acumulada es constante por tramos. Nótese también que $P\left[W \leq q_{\alpha}(x) \mid X=x\right]$ no necesariamente es igual a $\alpha$ (como lo es cuando $W$ es una variable aleatoria continua), por lo que es interesante estimar el verdadero nivel de servicio correspondiente a $q_{0.9}(x)$.

En el Cuadro 3 se reportan las estimaciones de los niveles de servicio tipo-I (probabilidad acumulada) para diferentes valores de $Q$, obtenidos luego de aplicar el algoritmo MP con $m=10^{6}$ repeticiones. A partir de los datos del cuadro se puede apreciar que el nivel de servicio correspondiente a $q_{0.9}(x)=4$ es aproximadamente $95.74 \%$

Cuadro 3. Niveles de Servicio Estimados para Diferentes Valores de $Q$ Usando MP con $m=10^{6}$

\begin{tabular}{ccc}
\hline $\begin{array}{c}\text { Inventario } \\
\text { inicial }(Q)\end{array}$ & $\begin{array}{c}\text { Nivel de servicia tipo-I } \\
\text { estimado }\left(\alpha_{1}\right)\end{array}$ & Ancho medio \\
\hline 1 & 0.460923 & $4.09 \mathrm{E}-04$ \\
2 & 0.722068 & $3.30 \mathrm{E}-04$ \\
3 & 0.881953 & $1.71 \mathrm{E}-04$ \\
4 & 0.957420 & $6.71 \mathrm{E}-05$ \\
5 & 0.986648 & $2.17 \mathrm{E}-05$ \\
6 & 0.996307 & $6.05 \mathrm{E}-06$ \\
7 & 0.999127 & $1.43 \mathrm{E}-06$ \\
8 & 0.999789 & $3.47 \mathrm{E}-07$ \\
9 & 0.999956 & $7.24 \mathrm{E}-08$ \\
10 & 0.999989 & $1.81 \mathrm{E}-08$ \\
11 & 0.999999 & $1.64 \mathrm{E}-09$ \\
12 & 1.000000 & $0.00 \mathrm{E}+00$ \\
\hline
\end{tabular}

Como puede apreciarse de los Cuadros 2 y 3, el número de repeticiones $m=10^{6}$ fue lo suficientemente grande para obtener errores de estimación pequeños para cada uno de los parámetros, lo cual podría no ocurrir para un número pequeño de repeticiones. Para ilustrar cómo este número afecta el cubrimiento y el ancho medio, se repitió el experimento de estimación $M=1,000$ veces para diferentes valores de $m, y$ se calcularon el cubrimiento empírico, el promedio y la desviación estándar de los anchos medios, el error cuadrático promedio y el sesgo para cada conjunto de experimentos. Para calcular estas medidas, se requiere de los valores teóricos (verdaderos) para los parámetros $r(x)$ y $q_{0.9}(x)$, por lo que se tomaron los valores $r(x)=1.82702$ y $q_{0.9}(x)=4$ obtenidos anteriormente. Los resultados se presentan en el Cuadro 4.

Como puede apreciarse de los datos del Cuadro 4, el cubrimiento empírico se aproxima al nominal de $90 \%$ a medida que el número de repeticiones crece, y para $m$ $=1,600$ ya se obtiene un cubrimiento aceptable. Nótese también que para $m=1,600$ se obtiene un sobre cubrimiento en la estimación de $q_{0.9}(x)$, lo que se explica por el hecho de que la variable de respuesta $W$ es discreta. Es conveniente notar que al incrementarse el tamaño de la muestra se aprecia una reducción del error de estimación, reduciéndose consistentemente el ancho medio, el error cuadrático promedio y el sesgo.

\section{Aplicación de la CMMC para incorporar una densidad a priori subjetiva}

Como se indicó en el segundo acápite de la segunda sección, cuando se usa la densidad a priori no informativa (gama) para el modelo con datos de censo, se pudo identificar que la distribución posterior es también gama, por lo que se pudo aplicar el algoritmo MP para la estimación de $r(x)$ y $q_{\alpha}(x)$. En esta sección se ilustra cómo se aplica la CMMC cuando se usa una densidad a priori subjetiva, para la cual no se ha podido identificar la familia de distribuciones a la que pertenece la distribución posterior.

Se consideró una densidad a priori uniforme en [ $a, b$ ], donde los valores de $a$ y $b$ son proporcionados por el usuario. La identificación de la distribución posterior con esta densidad a priori no es tarea fácil, por lo que se justifica el uso del algoritmo CMMC en este caso. Para la implementación del algoritmo, se consideró la 
Cuadro 4. Desempeño de MP con Base en $M=1,000$ Experimentos para Diferentes Valores de $m$

\begin{tabular}{|c|c|c|c|c|c|c|}
\hline \multirow{2}{*}{$\begin{array}{l}\text { Número de } \\
\text { repeticiones }\end{array}$} & \multirow{2}{*}{$\begin{array}{l}\text { Parámetro } \\
\text { estimado }\end{array}$} & \multirow{2}{*}{$\begin{array}{l}\text { Cubrimiento } \\
\text { empírico }\end{array}$} & \multicolumn{2}{|c|}{ Ancho medio } & \multirow{2}{*}{$\begin{array}{c}\text { Error } \\
\text { cuadrático } \\
\text { promedio }\end{array}$} & \multirow[b]{2}{*}{ Sesgo } \\
\hline & & & Promedio & $\begin{array}{l}\text { Desviación } \\
\text { estándar }\end{array}$ & & \\
\hline \multirow{2}{*}{$m=100$} & $r(x)$ & 0.878 & 0.2269 & 0.0187 & 0.0211 & 0.0064 \\
\hline & $q_{0.9}(x)$ & 0.679 & 0.5585 & 0.2467 & 0.3400 & -0.3340 \\
\hline \multirow{2}{*}{$m=400$} & $r(x)$ & 0.898 & 0.1139 & 0.0045 & 0.0047 & 0.0022 \\
\hline & $q_{0.9}(x)$ & 0.869 & 0.3360 & 0.2348 & 0.1310 & -0.1310 \\
\hline \multirow{2}{*}{$m=1,600$} & $r(x)$ & 0.904 & 0.0570 & 0.0011 & 0.0012 & 0.0003 \\
\hline & $q_{0,9}(x)$ & 0.987 & 0.1295 & 0.2191 & 0.0130 & -0.0130 \\
\hline
\end{tabular}

distribución de muestreo $q(\theta)$ que resulta de aplicar el procedimiento sugerido al final de la primera sección, como se explica a continuación.

Como $p(\theta)=1$, para $a<\theta<b$, sigue de (1) y (17) que:

$$
\begin{aligned}
& L_{n}(\theta)=\log [p(\theta \mid x)]= \\
& \sum_{i=1}^{p} \sum_{j=1}^{k_{i}} x_{i j} \log (\theta)-\theta \sum_{i=1}^{p} k_{i}-\log \left(\coprod_{i=1}^{p} k_{j=1}^{k_{i}}\right)
\end{aligned}
$$

por lo que

$$
\mu_{n}=\frac{\sum_{i=1}^{p} \sum_{j=1}^{k_{i}} x_{i j}}{\sum_{i=1}^{p} k_{i}}
$$

Por otro lado, como $L_{n}{ }^{\prime \prime}(\theta)=-\theta^{-2} \sum_{i=1}^{p} \sum_{j=1}^{k_{i}} x_{i j}$, se obtiene la varianza:

$$
\sigma^{2}=\left[-L_{n}{ }^{\prime \prime}\left(\mu_{n}\right)\right]^{-1}=\frac{\mu_{n}^{2}}{\sum_{i=1}^{p} \sum_{j=1}^{k_{i}} x_{i j}}
$$

Finalmente, considerando que $p(\theta \mid x)$ es cero cuando $\theta<a \circ \theta>b$, se tomó $q(\theta)$ igual a la densidad correspondiente a la distribución $N\left(\mu_{n}, \sigma^{2}\right)$ truncada en $[a, b]$, donde $\mu_{n}$ y $\sigma^{2}$ están definidas en (20) y (21), respectivamente.

Usando la densidad $q(\theta)$ así definida, se implementó el algoritmo CMMC para pronosticar la demanda $W$ definida en (14). Los parámetros de la densidad a priori se fijaron en $a=0$ y $b=0.02$ para $t_{0}=0.5$ y $k=500$. Los resultados de la estimación de $r(x)$ y $q_{0.9}(x)$ usando $m=10^{6}$ repeticiones se resumen en el Cuadro 5.

Cuadro 5. Estimaciones Obtenidas Usando la CMMC con $m=10^{6}$ Repeticiones

\begin{tabular}{ccc}
\hline Parámetro & Estimación por simulación & Ancho medio \\
\hline$r(x)$ & $\hat{r}_{M C}(x)=1.86013$ & 0.00254 \\
$q_{0.9}(x)$ & $\hat{q}_{0.9}^{M C}(x)=4$ & 0.0 \\
\hline
\end{tabular}

Como se aprecia de los Cuadros 2 y 5, los estimadores obtenidos con la densidad a priori subjetiva (uniforme) y con la densidad a priori objetiva (no informativa) son muy parecidas (diferencia menor de dos cifras decimales en el caso de $r(x)$ y exactamente iguales en el caso de $q_{0.9}(x)$ ). Este resultado no es sorprendente si se toma en cuenta (del Cuadro 1) que el tamaño de muestra ( $\sum_{i=1}^{p} k_{i}=4,584$ ) es grande, y en este caso la distribución posterior tiene poca influencia de la distribución a priori.

Otra interesante consecuencia de disponer de un tamaño de muestra grande es que se reduce la incertidumbre paramétrica, y la varianza $\sigma_{W}^{2}$, definida en (9), está dominada por la varianza estocástica $\sigma_{s}^{2}$. Para ilustrar esta propiedad, nótese que en este modelo se tiene que $r_{l}(\theta)=\sigma^{2}(\theta)=k t_{0} \theta$, por lo que, bajo la densidad a priori objetiva (gama) se 
puede verificar fácilmente que $\sigma_{p}^{2}=\left(k t_{0}\right)^{2} \beta_{1} \beta_{2}^{-2}$, y $\sigma_{s}^{2}=k t_{0} \beta_{1} \beta_{2}^{-1}$, donde $\beta_{1}=\sum_{i=1}^{p} \sum_{j=1}^{k_{i}} x_{i j}$ y $\beta_{2}=\sum_{i=1}^{p} k_{i}$. Estos resultados permiten verificar que, para los datos del Cuadro 1, la varianza estocástica $\sigma^{2}$ representa el $94.82 \%$ de la varianza total $\sigma_{w}^{2}$.

Para proporcionar un ejemplo de cómo estos métodos bayesianos pueden desempeñarse de otra forma, dependiendo del conjunto de datos disponible, se simuló un conjunto diferente de datos para el mismo problema de estimación. El nuevo conjunto de datos se presenta en el Cuadro 6, y fue obtenido considerando un número fijo de máquinas en operación $k_{i}=10$, $i=1,2, \ldots, 12$, y fallas distribuidas según una distribución de Poisson con tasa de fallas de $\theta=1$ por mes.

Cuadro 6. Datos Simulados para 10 Máquinas y Tasa de Fallas $\theta=1$

\begin{tabular}{lcc}
\hline \multicolumn{1}{c}{ Mes $(i)$} & $\begin{array}{c}\text { Máquinas en } \\
\text { operación }\left(k_{i}\right)\end{array}$ & Fallas $\left(\sum_{j=1}^{k_{i}} x_{i j}\right)$ \\
\hline Enero (1) & 10 & 8 \\
Febrero (2) & 10 & 13 \\
Marzo (3) & 10 & 13 \\
Abril (4) & 10 & 14 \\
Mayo (5) & 10 & 9 \\
Junio (6) & 10 & 14 \\
Julio (7) & 10 & 12 \\
Agosto (8) & 10 & 8 \\
Septiembre (9) & 10 & 11 \\
Octubre (10) & 10 & 13 \\
Noviembre (11) & 10 & 13 \\
Diciembre (12) & 10 & 16 \\
Total & $\mathbf{1 2 0}$ & $\mathbf{1 4 4}$ \\
\hline
\end{tabular}

Las estimaciones de $r(x)$ y $q_{0.9}(x)$ usando $m=10^{6}$ repeticiones, $k=10$ y $t_{0}=1$ se resumen en el Cuadro 7 para los métodos MP y CMMC. Para el algoritmo MP se consideró la densidad a priori objetiva (gama), y para el algoritmo CMMC se consideró la densidad a priori uniforme en $[0.8,1.2]$.
Cuadro 7. Estimación de Parámetros Usando MP y CMMC con $m=10^{6}$ Repeticiones

\begin{tabular}{llr}
\hline Método & \multicolumn{1}{c}{$\begin{array}{c}\text { Estimación por } \\
\text { simulación }\end{array}$} & \multicolumn{1}{c}{$\begin{array}{c}\text { Ancho } \\
\text { medio }\end{array}$} \\
\hline $\begin{array}{l}\text { Muestreo } \\
\text { posterior }\end{array}$ & $\hat{r}(x)=12.0449$ & 0.00594 \\
$\begin{array}{l}\text { Cadena de } \\
\text { Markov } \\
\text { Monte Carlo }\end{array}$ & $\hat{q}_{0.9}(x)=17$ & 0.0 \\
\hline
\end{tabular}

En el Cuadro 7 se resumen los resultados de las estimaciones de $r(x)$ y $q_{0.9}(x)$ usando densidades a priori diferentes. En el caso de MP, la densidad a priori no informativa asume poco conocimiento a priori sobre el valor del parámetro $\Theta$. Por otro lado, para el caso de la CMMC, la densidad a priori uniforme en $[0.8,1.2]$ proporciona mayor información sobre $\Theta$. Como puede observarse en el cuadro mencionado, la influencia de la densidad a priori en el valor de los parámetros es mucho más grande que la observada con los datos del Cuadro 1. En la Figura 1 se presentan las gráficas de la función de distribución acumulada de $W$ obtenidas bajo las dos diferentes densidades a priori, donde se puede apreciar con mayor claridad la diferencia. Nótese que la distribución de $W$ bajo la densidad a priori no informativa tiene una varianza más grande, lo que refleja una mayor incertidumbre en el pronóstico.

\section{CONCLUSIONES Y DIRECCIONES PARA INVESTIGACIONES FUTURAS}

Este artículo ilustra cómo se puede combinar un modelo de simulación con técnicas de estimación bayesiana para estimar parámetros de pronóstico en problemas de administración de inventarios de repuestos. La ventaja de usar la simulación como una técnica de pronóstico radica en el hecho de que se pueden utilizar modelos muy complejos, que incorporan información detallada del sistema, con la finalidad de producir pronósticos más confiables. 


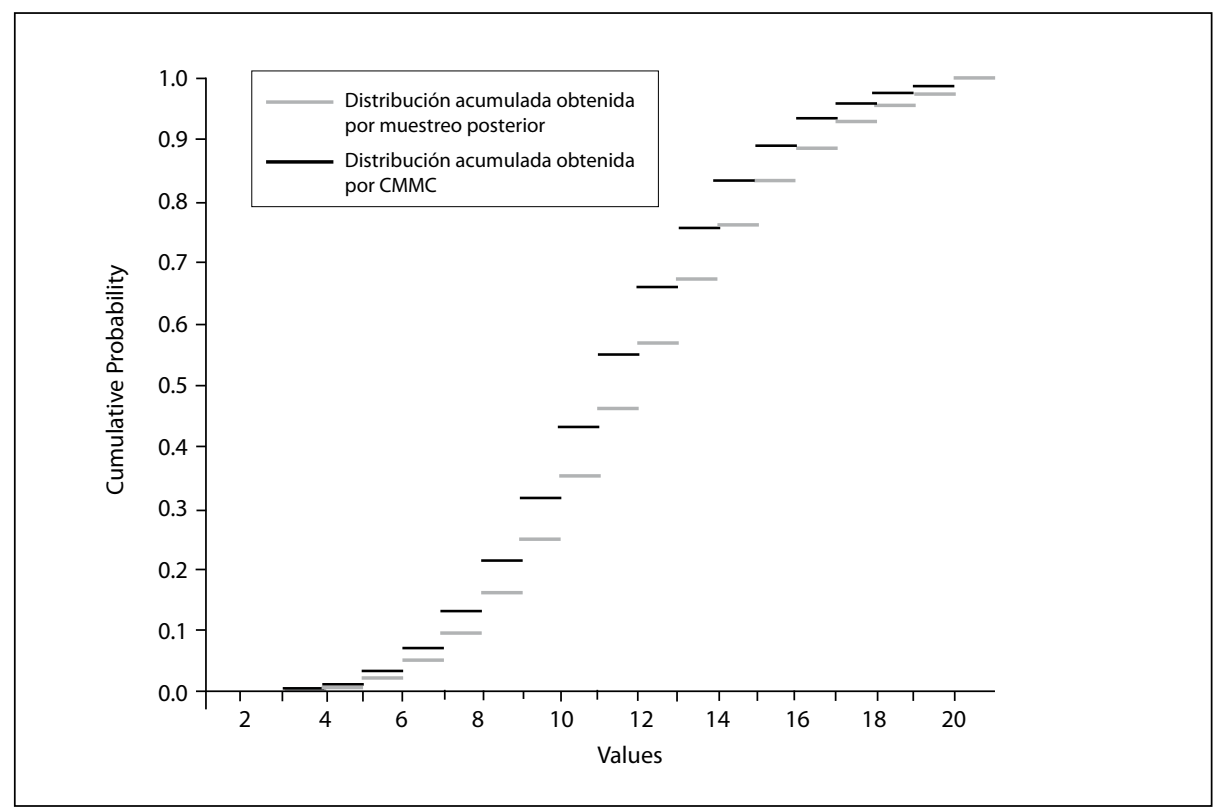

Figura 1. Distribución acumulada de la variable de respuesta usando datos de fallas simulados

Se han ilustrado las aplicaciones potenciales de los métodos de muestreo posterior y de la cadena de Markov Monte Carlo como técnicas para construir pronósticos utilizando simulación estocástica. El método de muestreo posterior (MP) requiere de un algoritmo para generar muestras de la función de densidad posterior $p(\theta \mid x)$, mientras que el método de la cadena de Markov Monte Carlo (CMMC) puede ser aplicado aun cuando no se haya identificado la familia de distribuciones a la que pertenece $p(\theta \mid x)$. La aplicación de estos métodos se ilustró proponiendo dos modelos sencillos para el pronóstico de fallas de repuestos; el primer modelo considera la existencia de datos de tiempos entre fallas sucesivas, mientras que el segundo considera datos de censo, y en ambos casos se utilizó un marco bayesiano para el pronóstico de la demanda de repuestos.

Se aplicó el modelo con datos de censo, utilizando datos reales de un distribuidor de autos y repuestos de México, D. F., y ambas metodologías, MP y CMMC, cuando fueron necesarias. Para aplicar MP se consideró la distribución de referencia (no informativa), mientras que para la CMMC se consideró una distribución uniforme (subjetiva). Los resultados de la aplicación de estos métodos han permitido establecer las siguientes conclusiones.

En primer lugar, el error de estimación (medido por el ancho medio), así como el sesgo y el error cuadrático promedio disminuyen a medida que crece el número de repeticiones $m$ del experimento de estimación por simulación. En consecuencia, desde el punto de vista de la aplicación, es importante establecer el número de repeticiones $m$ que posibilita obtener el grado de error permitido, y el cálculo del correspondiente ancho medio es importante para medir la magnitud del error de estimación de la simulación.

En segundo lugar, los resultados obtenidos usando datos reales ilustran cómo un tamaño grande de la muestra permite que la distribución posterior tenga poca influencia de la distribución a priori. El pronóstico obtenido con la densidad a priori objetiva resultó muy similar al que se obtiene con una distribución más informativa, debido a que, en este caso, la densidad a priori tiene poca influencia en la distribución posterior. Para ilustrar esta propiedad, se simuló otro conjunto de datos con un tamaño de muestra más pequeño y una tasa de fallas más grande. En este caso, la influencia de 
la densidad a priori se hizo más evidente, obteniéndose pronósticos considerablemente diferentes.

Finalmente, la relevancia de las metodologías ilustradas en este artículo depende de la habilidad del modelo propuesto para imitar el sistema real, la que está relacionada con una adecuada selección de los parámetros para los cuales existe información muestral, así como de las relaciones entre los componentes aleatorios que generan la incertidumbre estocástica. En esta dirección, los modelos propuestos en la segunda sección pueden modificarse para reflejar mejor el sistema en estudio, por ejemplo, asumiendo una fami- lia diferente de distribuciones para los tiempos entre fallas del repuesto. Similarmente, estos métodos que combinan la simulación con un enfoque bayesiano de pronóstico podrían ser apropiados para resolver problemas de pronóstico en cadenas de suministro (véase, por ejemplo, Kalchschmidt et ál., 2006).

Es conveniente remarcar que, si bien en este artículo se utilizaron modelos relativamente sencillos, el mayor potencial de las metodologías ilustradas radica en su posible aplicación con modelos de simulación que incluyen información detallada sobre el proceso de generación de la demanda.

\section{REFERENCIAS}

Alba, E. de, \& Mendoza, M. (2007). Bayesian forecasting methods for short time series. Foresight, 8, 41-44.

Asmussen, S., \& Glynn, P. W. (2007). Stochastic simulation algorithms and analysis. New York: Springer.

Bartezzaghi, E., Verganti, R., \& Zotteri, G. (1999). A simulation framework for forecasting uncertain lumpy demand. International. Journal of Production Economics, 59, 499-510.

Berger, J. O., Bernardo, J. M., \& Sun, D. (2009). The formal definition of reference priors. Annals of Statistics, $37(2), 905-938$.

Bernardo, J. M., \& Smith, A. F. M. (2000). Bayesian theory. Chichester: John Wiley.

Caniato, F., Kalchschmidt, M., Ronchi, E., Veganti, R., \& Zotteri, G. (2005). Clustering customers to forecast demand. Production Planning \& Control, 16, 32-43.

Cheng, R. C. H., \& Holland, W. (2004). Calculation of confidence intervals for simulation output. ACM Transactions on Modeling and Computer Simulation, 14(4), 344-362.

Chopra, S., \& Meindl, P. (2004). Supply chain management.

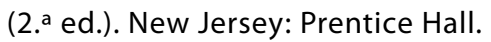

Chung, K. L. (1974). A course in probability theory. (2. a ed.). San Diego: Academic Press.

Croston, J. D. (1972). Forecasting and stock control for intermediate demands. Operational Research Quarterly, 23, 289-303.
Kalchschmidt, M., Verganti, R., \& Zotteri, G. (2006). Forecasting demand from heterogeneous customers. International Journal of Operations \& Production Management, 26(6), 619-638.

Makridakis, S., Wheelwright, S. C., \& Hyndman, R. J. (1998). Forecasting: Methods and applications. (3. a ed.). New York: John Wiley.

Muñoz, D. F. (2009). Pronósticos bayesianos usando simulación estocástica. Cuadernos de Difusión. 14(26), 7-26.

Rao, A. V. (1973). A comment on forecasting and stock control for intermediate demands. Operational Research Quarterly, 24, 639-640.

Schmeiser, B. (1982). Batch size effects in the analysis of simulation output. Operations Research, 30(3), 556-568.

Serfling, R. J. (1980). Approximation theorems of mathematical statistics. New York: John Wiley.

Syntetos, A. A., \& Boylan, J. E. (2001). On the bias of intermittent demand estimates. International. Journal of Production Economics, 71, 457-466.

Wacker, J. G., \& Sprague, L. G. (1998). Forecasting accuracy: comparing the relative effectiveness of practices between seven developed countries. Journal of Operations Management, 16, 271-290.

Willemain, T. R., Smart, C. N., \& Schwarz, H. F. (2004). A new approach to forecasting intermittent demand for service parts inventories. International Journal of Forecasting, 20, 375-387. 
Zotteri, G., \& Kalchschmidt, M. (2007a). Forecasting practices: Empirical evidence and a framework for research. International. Journal of Production Economics, 108, 84-99.
Zotteri, G., \& Kalchschmidt, M. (2007b). A model for selecting the appropriate level of aggregation in forecasting processes. International Journal of Production Economics, 108, 74-83. 